\title{
Smart Sensing Technologies and Their Applications in Civil Infrastructures 2016
}

\author{
Hong-Hu Zhu, ${ }^{1}$ Fei Dai, ${ }^{2}$ Zhenhua Zhu, ${ }^{3}$ Tuan Guo, ${ }^{4}$ and Xiao-Wei Ye ${ }^{5}$ \\ ${ }^{1}$ School of Earth Sciences and Engineering, Nanjing University, Nanjing 210023, China \\ ${ }^{2}$ Department of Civil and Environmental Engineering, West Virginia University, Morgantown, WV 26506, USA \\ ${ }^{3}$ Department of Building, Civil and Environmental Engineering, Concordia University, Montreal, QC, Canada H3G $1 M 8$ \\ ${ }^{4}$ Institute of Photonics Technology, Jinan University, Guangzhou 510632, China \\ ${ }^{5}$ Department of Civil Engineering, Zhejiang University, Hangzhou 310058, China
}

Correspondence should be addressed to Hong-Hu Zhu; zhh@nju.edu.cn

Received 31 October 2016; Accepted 1 November 2016

Copyright (c) 2016 Hong-Hu Zhu et al. This is an open access article distributed under the Creative Commons Attribution License, which permits unrestricted use, distribution, and reproduction in any medium, provided the original work is properly cited.

In recent years, numerous civil infrastructures have been built in metropolitan areas all over the world. The performance of these infrastructures during construction, operation, maintenance, and upgrading is a major concern for the society. The use of smart sensing technologies for structural health monitoring, nowadays, has attracted much attention due to their exceptional benefits [1]. These technologies have developed rapidly and some have found widespread applications in civil and geotechnical engineering practices, such as fiber optic sensing (FOS), time domain reflectometry (TDR), microelectromechanical system (MEMS), particle image velocimetry (PIV), acoustic emission (AE), and wireless sensor network (WSN).

This special issue is devoted to the research area of smart sensing technologies and their applications in civil infrastructures. The topics cover various aspects including novel smart sensing mechanisms and devices for civil infrastructures, applications of smart sensors and sensing systems in civil infrastructures, in-field implementation of structural health monitoring and diagnosis systems, supporting technologies and methodologies in monitoring civil infrastructures, and typical case study of sensing applications and civil infrastructures monitoring.

The issue contains two papers dealing with the applications of Fiber Bragg Grating (FBG) to structural health monitoring. Based on this technology, a quasi-distributed fiber optic sensing array can be established to perform accurate strain and temperature measurements $[2,3]$. In the research article titled "Fiber Bragg Grating Sensors-Based In Situ Monitoring and Safety Assessment of Loess Tunnel," J. Lai et al. present an investigation of the feasibility of tunnel monitoring using the quasi-distributed FBG sensors. The paper by P. Wang et al. introduces the application of FBG to measure the longitudinal temperature forces of seamless rails. Laboratory tests and field monitoring were conducted to evaluate the measuring accuracy. Besides FBG, another popular FOS technology is the fully distributed Brillouin optical time domain reflectometry (BOTDR), which enables the measurement of strain and temperature profiles along a single-mode optical fiber $[4,5]$. Y. Sun et al. successfully utilized the newly developed BOTDR inclinometers to monitor the deformation of Majiagou Landslide. In their paper, the working principle and field performance of this inclinometer are presented.

In recent years, TDR has been used to measure shear deformation in boreholes [6] or moisture content of geomaterials [7]. The paper of $\mathrm{D}$. Ling et al. introduced an experimental study on the relationship between dielectric constant of TDR coaxial cables and water contents of rocksoil mixture.

As a novel visual sensing technology, PIV has great potential in deformation monitoring of physical models [8]. In the paper of G. Chen et al., the acceleration characteristics of a rock slide during laboratory testing are studied by using this technology. The failure mechanism of the rock slope model is analyzed based on the monitoring results of 
displacement fields. The paper of K.-W. Min et al. introduces a standalone vision sensing system for pseudodynamic testing of tuned liquid column dampers. X. W. Ye et al. present a critical review of the recent development of machine visionbased structural health monitoring. The basic methodologies and practical applications of this technology are discussed in detail.

Nowadays, Fiber Reinforced Polymer (FRP) is a widely used material for structural upgrades and ground improvement [9]. The field performance of this material should be explored carefully. In the paper of R. Krzywon et al., a self-monitoring strengthening system is developed based on carbon fiber laminate. Several trials have been conducted to evaluate the performance of this system.

We hope that this special issue would shed light on development and applications of smart sensing technologies in civil infrastructures and attract attention by the scientific community and civil engineering industry to pursue further investigations leading to the rapid implementation of these sensing technologies.

\section{Acknowledgments}

The guest editors of this special issue would like to express their sincere gratitude to all the authors for their informative contributions and the anonymous reviewers for their support and constructive critiques in making this special issue possible.

$$
\begin{array}{r}
\text { Hong-Hu Zhu } \\
\text { Fei Dai } \\
\text { Zhenhua Zhu } \\
\text { Tuan Guo } \\
\text { Xiao-Wei Ye }
\end{array}
$$

\section{References}

[1] K. Soga and J. Schooling, "Infrastructure sensing," Interface Focus, vol. 6, no. 4, Article ID 20160023, 2016.

[2] T. Guo, Q. Zhao, Q. Dou et al., "Temperature-insensitive fiber Bragg grating liquid-level sensor based on bending cantilever beam," IEEE Photonics Technology Letters, vol. 17, no. 11, pp. 2400-2402, 2005.

[3] H.-H. Zhu, A. N. L. Ho, J.-H. Yin, H. W. Sun, H.-F. Pei, and C.Y. Hong, "An optical fibre monitoring system for evaluating the performance of a soil nailed slope," Smart Structures \& Systems, vol. 9, no. 5, pp. 393-410, 2012.

[4] L. Luo, B. Li, Y. Yu, X. Xu, K. Soga, and J. Yan, "Time and frequency localized pulse shape for resolution enhancement in STFT-BOTDR," Journal of Sensors, vol. 2016, Article ID 3204130, 10 pages, 2016.

[5] W. Suo, Y. Lu, B. Shi, H. Zhu, G. Wei, and H. Jiang, "Development and application of a fixed-point fiber-optic sensing cable for ground fissure monitoring," Journal of Civil Structural Health Monitoring, vol. 6, no. 4, pp. 715-724, 2016.

[6] A. K. Leung and C. W. Ng, "Field investigation of deformation characteristics and stress mobilisation of a soil slope," Landslides, vol. 13, no. 2, pp. 229-240, 2016.

[7] W.-T. Hong, Y.-S. Jung, J.-S. Lee, and Y.-H. Byun, “Development and application of TDR penetrometer for evaluation of soil water content of subsoil," Journal of the Korean Geotechnical Society, vol. 31, no. 2, pp. 39-46, 2015.

[8] C.-C. Zhang, H.-H. Zhu, and B. Shi, "Role of the interface between distributed fibre optic strain sensor and soil in ground deformation measurement," Scientific Reports, vol. 6, Article ID 36469, 2016.

[9] J. R. Murugadoss, B. J. Lee, J. W. Bang, G. G. Prabhu, and Y. Y. Kim, "Performance analysis of CFRP composite strips confined RC columns under axial compression," Advances in Materials Science and Engineering, vol. 2015, Article ID 170295, 18 pages, 2015. 


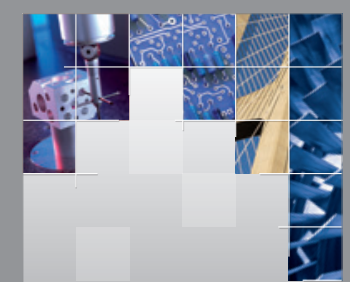

\section{Enfincering}
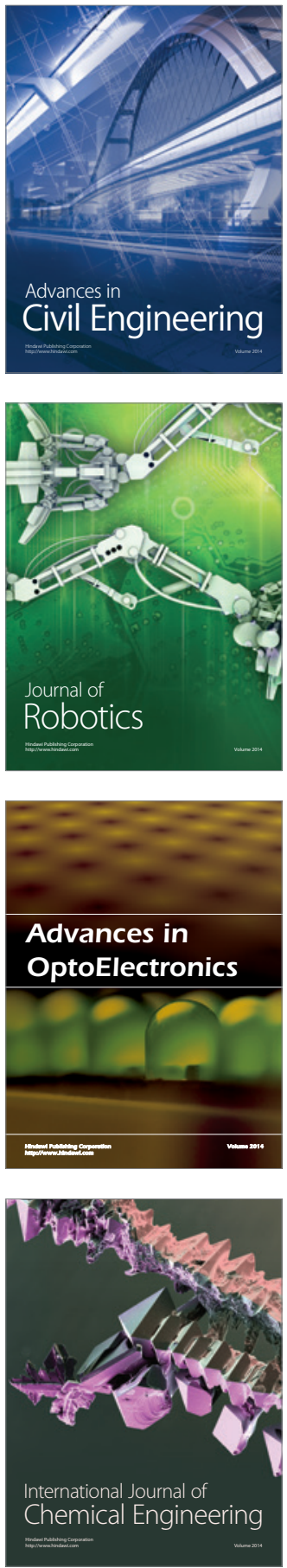

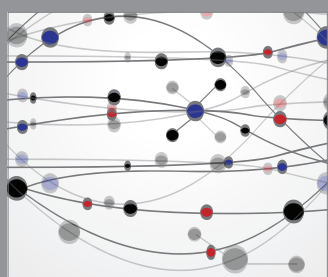

The Scientific World Journal

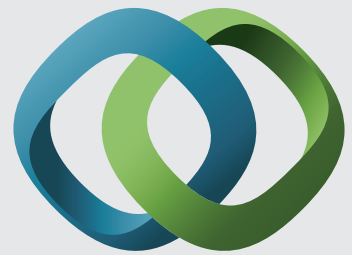

\section{Hindawi}

Submit your manuscripts at

http://www.hindawi.com
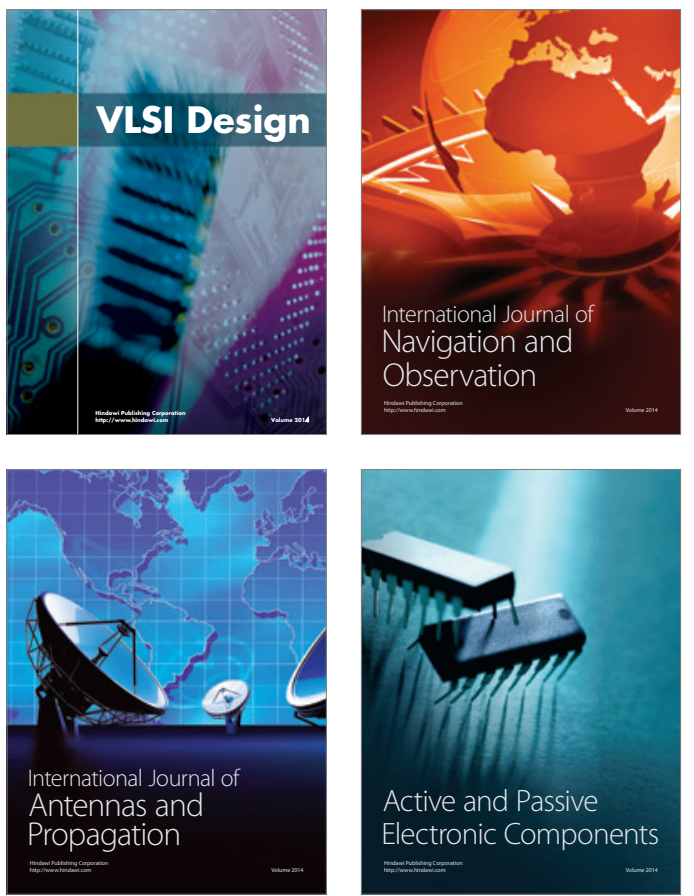
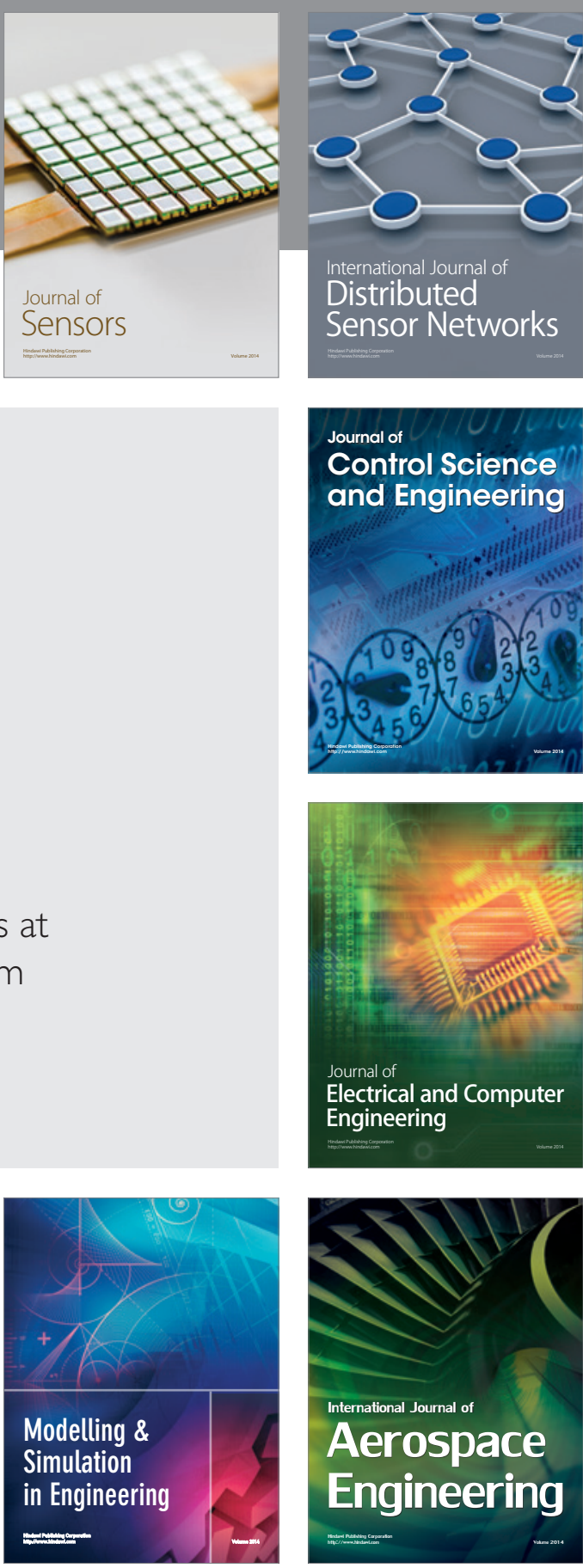

International Journal of

Distributed

Sensor Networks

Journal of

Control Science

and Engineering
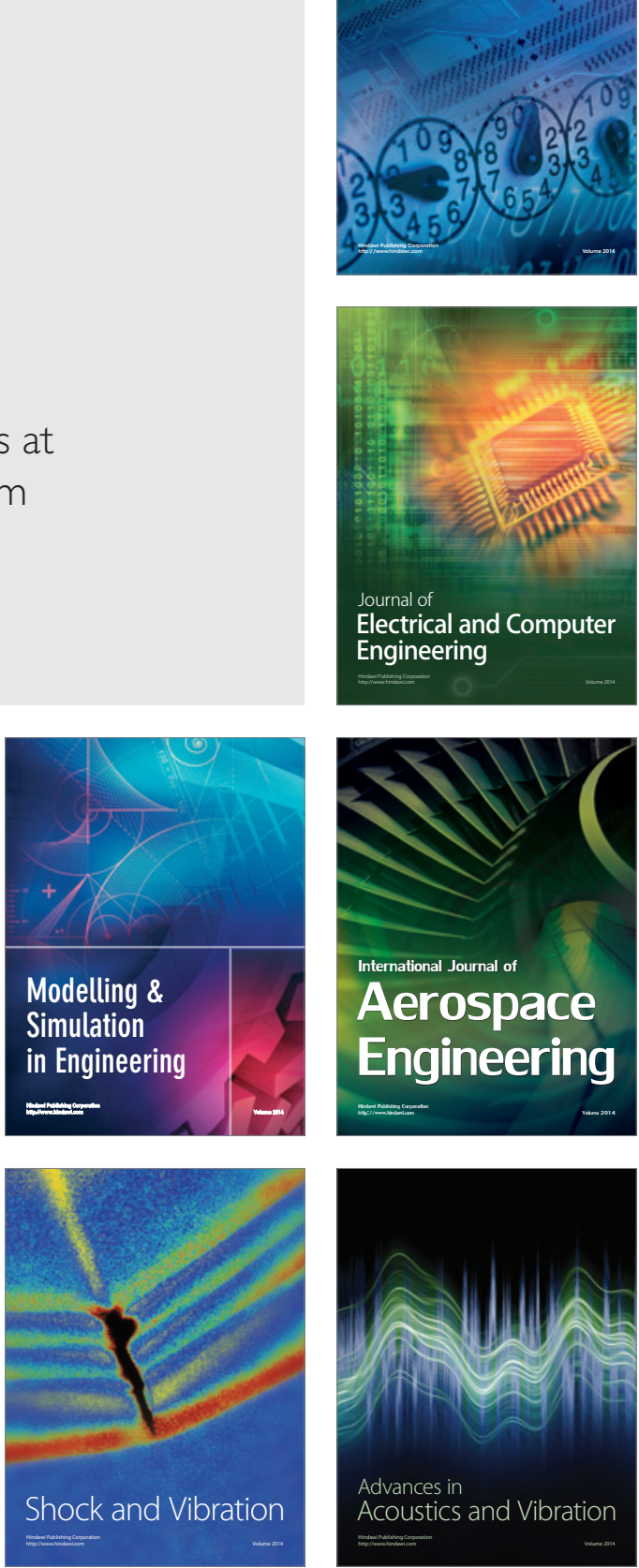